\title{
Increasing the accuracy of 3D EEG implantations
}

\author{
Roman Rodionov, PhD, ${ }^{1-3}$ Aidan O'Keeffe, PhD, ${ }^{4}$ Mark Nowell, PhD, FRCS(SN), ${ }^{1-3}$ \\ Michele Rizzi, MD, ${ }^{2,3,5}$ Vejay N. Vakharia, MD, ${ }^{1-3}$ Victoria Wykes, MD, PhD, ${ }^{2}$ \\ Sofia H. Eriksson, MD, PhD, ${ }^{1-3}$ Anna Miserocchi, MD, ${ }^{1,2}$ Andrew W. McEvoy, FRCS(SN), ${ }^{1,2}$ \\ Sebastien Ourselin, PhD, ${ }^{6,7}$ and John S. Duncan, MD, FRCP1-3
}

${ }^{1} \mathrm{UCL}$ Queen Square Institute of Neurology, Department of Clinical and Experimental Epilepsy, University College London; ${ }^{2}$ National Hospital for Neurology and Neurosurgery, London; ${ }^{3}$ Epilepsy Society, Chalfont St. Peter, Buckinghamshire;

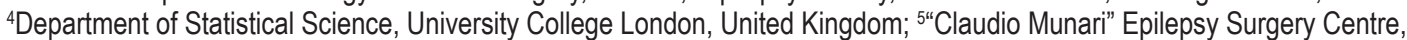
Ospedale Niguarda Ca' Granda, Milan, Italy; ${ }^{6}$ Centre for Medical Imaging Computing, University College London; and ${ }^{7}$ School of Biomedical Engineering and Imaging Sciences, Kings College London, United Kingdom

OBJECTIVE The accuracy of stereoelectroencephalography (SEEG) electrode implantation is an important factor in maximizing its safety. The authors established a quality assurance (QA) process to aid advances in implantation accuracy.

METHODS The accuracy of three consecutive modifications of a frameless implantation technique was quantified in three cohorts comprising 22, 8, and 23 consecutive patients. The modifications of the technique aimed to increase accuracy of the bolt placement.

RESULTS The lateral shift of the axis of the implanted bolt at the level of the planned entry point was reduced from a mean of $3.0 \pm 1.6 \mathrm{~mm}$ to $1.4 \pm 0.8 \mathrm{~mm}$. The lateral shift of the axis of the implanted bolt at the level of the planned target point was reduced from a mean of $3.8 \pm 2.5 \mathrm{~mm}$ to $1.6 \pm 0.9 \mathrm{~mm}$.

CONCLUSIONS This QA framework helped to isolate and quantify the factors introducing inaccuracy in SEEG implantation, and to monitor ongoing accuracy and the effect of technique modifications.

https://thejns.org/doi/abs/10.3171/2019.2.JNS183313

KEYWORDS implantation; accuracy; quality assurance; SEEG; epilepsy

$\mathrm{A}$ PPROXIMATELY $25 \%$ of individuals with refractory focal epilepsy who may benefit from epilepsy surgery require intracranial electrodes to localize and delineate the epileptogenic zone. The electrode implantation follows the formulation of a strategy devised by the multidisciplinary team.

The safety of stereoelectroencephalography (SEEG) depends on the planned trajectories and the accuracy of implementation of the plan. With a highly accurate implantation technique, plans that may be considered too risky if implantation is not accurate may still be performed. The safety of an SEEG implantation plan has objective and subjective components in its interpretation by different neurosurgeons. ${ }^{9}$ The accuracy of SEEG implantation is entirely objective, as it is a geometrical concept based on avoidance of damaging vascular structures.
Recent advances in SEEG implantation techniques, such as robotic guidance, ${ }^{1,3}$ allow highly accurate implementation of the planned trajectories, bringing the accuracy to the level of frame-based techniques. ${ }^{6,8}$ The present standard method of measuring implantation accuracy is the distance between planned and implemented trajectories at the level of the entry point (EP) and target point (TP). A limited number of surgical groups ${ }^{2,7,8}$ have reported lateral deviation of the implanted tip of the electrode from the planned trajectory. The lateral shift allows disentangling of the depth insertion error, which was noted but not measured in two studies. ${ }^{5,8}$ Errors of depth insertion are recognized ${ }^{10}$ and may exceed $10 \mathrm{~mm} .{ }^{11}$

Another factor that may affect the reported integral accuracy of the implantation is bending of the electrode during insertion. This effect is well recognized ${ }^{1,3}$ but has not

ABBREVIATIONS EP = entry point; $i E P=$ implemented EP; IQR = interquartile range; $i T P=$ distal contact of the implanted electrode; $M 1, M 2, M 3=$ method 1, method 2, method 3; NHNN = National Hospital for Neurology and Neurosurgery; PTP = planned TP; QA = quality assurance; SEEG = stereoelectroencephalography; TP = target point.

SUBMITTED November 27, 2018. ACCEPTED February 12, 2019.

INCLUDE WHEN CITING Published online May 17, 2019; DOI: 10.3171/2019.2.JNS183313. 
been systematically quantified. Depth insertion errors and electrode bending contribute to the integral accuracy of the procedure, measured as the distance between the tip of the implanted electrode and the planned TP (pTP).

Accuracy is typically reported using summary statistics (mean/median, confidence intervals/interquartile range [IQR]). Few studies report outliers and maximum errors ${ }^{3,11}$ which, even with very accurate techniques (such as robot guidance and a patient-specific frame), may exceed $5 \mathrm{~mm}$ and $10 \mathrm{~mm}$, respectively, for the integral accuracy at the TP. Understanding the factors contributing to inaccuracy is important for the safety of implantation surgery and for minimizing risk.

In general, the level of spatial accuracy needed to test neurophysiological hypotheses about seizure onset is less than the spatial accuracy of the SEEG implantation techniques. Consideration of the greatest deviations from the $\mathrm{TP}$ is important if the anatomical target-e.g., amygdala, hippocampus, and subependymal heterotopic nodules-is missed and the neurophysiological hypothesis cannot be tested.

Interpretations of reports of implantation accuracy are limited because 1) the published metrics do not separate the accuracy of implanting the guiding bolt from the accuracy of the electrode insertion, and 2) different surgical centers use electrode-related hardware (guiding bolt, stylet, electrode) that have different geometric and physical characteristics, which affect the observed deviation of the inserted electrode from the axis of the guiding bolt. We developed a quality assurance (QA) process to monitor and improve our frameless implantation techniques, ${ }^{7}$ and demonstrate the detailed quantification of accuracy of two stages of the procedure: bolt placement and electrode insertion.

\section{Methods}

\section{Evolution of the SEEG Electrode Implantation Technique}

The details of the frameless SEEG implantation technique that we use at the National Hospital for Neurology and Neurosurgery (NHNN) are described elsewhere? Briefly, an articulated Vertek arm (Medtronic Ltd) is used to align the guiding tube with the planned trajectory; a hole in the skull is made by drilling along the direction of the guiding tube; a guiding bolt is inserted into the skull; and a rigid stylet is inserted through the guiding bolt to prepare the path for a more flexible electrode that is inserted once the stylet is removed. This technique is further referred to as method 1 (M1).

Our SEEG implantation technique has evolved through the following stages. M1 was practiced from July 2012 until May 2015. Key features of this method included scalp fiducials to register the patient and the neuronavigation system, and "target guidance" alignment mode of neuronavigation software when only the TP is shown on the screen during the alignment process. Method 2 (M2) was practiced from June 2015 until September 2015. Key features of M2 included bone fiducials, and "target guidance" alignment mode that enables aligning only the TP without visualizing the $\mathrm{EP}$ on the screen of the neuronavigation system. Method 3 (M3), which has been practiced from
October 2015 until present, included bone fiducials, as well as "trajectory guidance" alignment mode (StealthStation S7 Cranial software, version 2.2.6 or higher, Medtronic) when both the EP and TP of the planned trajectory are presented on the screen of the neuronavigation system, allowing their simultaneous alignment with the planned trajectory.

The complete QA data collection process was established in June 2015, including the variations of the surgical technique M2 and M3. Data for the M1 stage were less comprehensive, omitting the measurements at the EP and the angle between the planned trajectory and the implanted bolt. ${ }^{7}$ We did not have a complete data set of measurements available for the initial technique (M1), and so we primarily focused on comparing techniques M2 and M3.

The modifications applied to the technique were focused on improving the accuracy of placing the bolt, as this is the single most important part of the surgical procedure that determines accuracy. The method of inserting the electrode through the bolt remained unchanged through the three stages.

\section{Patients}

The M1 cohort consisted of 22 patients (187 total electrodes). ${ }^{7}$ Group M2 comprised 8 patients (83 total electrodes), and group M3 comprised 23 patients (202 total electrodes).

\section{Model of Implantation Accuracy}

The quantification of the accuracy of independent stages of the procedure is at the core of the detailed QA. The accuracy of placing the bolt is quantified by 3 parameters (Fig. 1):

- P1, lateral shift of the axis of the EP of the implanted bolt into the scalp (implemented EP, or iEP) from the planned trajectory (measured in $\mathrm{mm}$ );

- P2, lateral shift of the pTP from the axis of the implanted bolt (measured in $\mathrm{mm}$ ); and

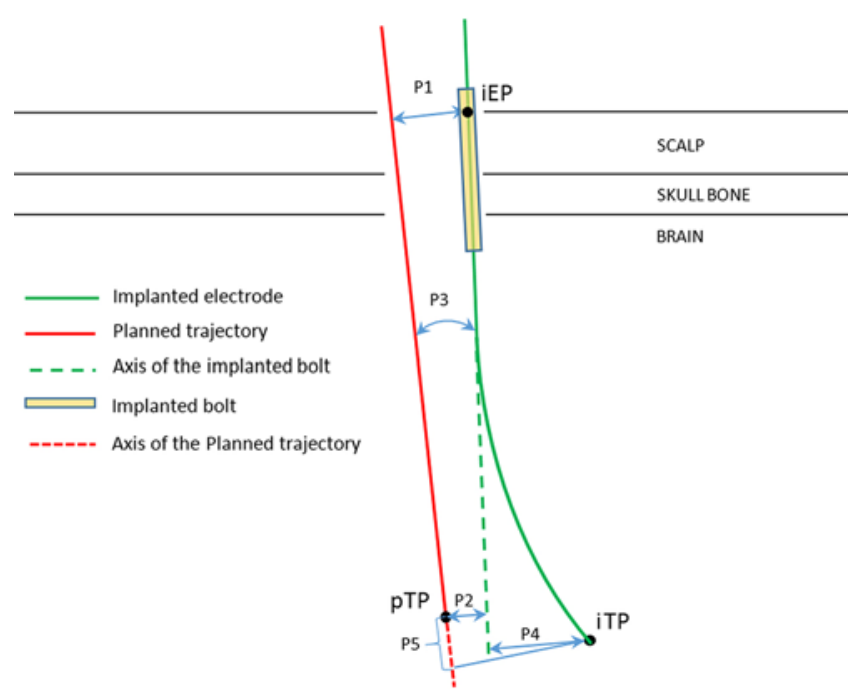

FIG. 1. The geometric model of implantation accuracy (parameters P1 to P5; see details in the text) shown using the projections of the planned trajectory and the implanted bolt. Figure is available in color online only. 
- P3, angle between the planned trajectory and the axis of the implanted bolt (measured in degrees). The lateral shift of a point is a distance along the perpendicular from the point to the line.

The accuracy of the electrode insertion is measured using 2 further parameters (Fig. 1):

- P4, electrode bending, the distance from the most distal contact of the implanted electrode (as visualized on the postimplantation CT image) to the axis of the implanted bolt when measured along the perpendicular to the axis of the bolt $(\mathrm{mm})$; and

- P5, depth deviation, the distance along the planned trajectory from the pTP to the point of its intersection with the perpendicular drawn from the distal contact of the implanted electrode (iTP, measured in $\mathrm{mm}$ ).

The distance between the pTP and iTP describes the integral accuracy of the placement of the electrode and is widely used to describe the accuracy of the technique. We hypothesized that the following parameters might influence the accuracy of the procedure.

- The duration of drilling was expected to affect the accuracy of the bolt placement. It was recorded for $16 \mathrm{pa}-$ tients (148 trajectories) from the M3 cohort. Prolonged drilling may introduce increased inaccuracy due to deviations of the surgeon's arm holding the drill. This could influence parameters P1, P2, and P3. The same surgeon performed drilling during all operations in this study.

- The angle between the planned trajectory and the skull surface may affect the accuracy of the bolt placement. Shallow entry angles increase the chances of the drill bit slipping at the start of drilling. The angle between the axis of the implanted bolt and the skull surface is a good approximation of this parameter. This angle was measured for all patients in the M3 cohort. The potentially affected parameters are expected to be P1, P2, and P3.

- The depth to which the rigid stylet is introduced is likely to affect the accuracy of inserting the electrode. This measurement was recorded in 14 patients (129 trajectories). The trajectories with the stylet inserted significantly short of the pTP are likely to show decreased accuracy of the tip of the electrode and are described by parameter $\mathrm{P} 4$.

A fixed-effects linear model was fitted to the data to test for dependency between the measured parameters (see Supplementary Material for the results).

\section{Workflow for Obtaining the QA Measurements}

\section{Using EpiNav Software (UCL)}

The implantation plan is developed using the gadolinium-enhanced T1-weighted MR image and exported in a format compatible with Cranial software of the neuronavigation system (StealthStation S7). The navigation CT image is acquired after placement of the scalp (M1 technique) or bone (M2 and M3 techniques) fiducials.

\section{Using StealthStation Cranial Software}

Both the implantation plan and the navigation CT image are loaded into the neuronavigation station and the implantation plan registered to the space of the navigation image. The implantation plan is reviewed, modified if required, and approved by the operating surgeon. The coordinates of the planned trajectories (pEP, pTP) are exported for QA analysis.

The postimplantation CT image showing the implanted electrodes is loaded onto the neuronavigation station and registered to the anatomical space of the navigation $\mathrm{CT}$ image. The following points are labeled to measure the parameters of the implanted bolt and the electrode: both ends on the implanted bolt on its axis; EP of the bolt axis into the scalp (iEP); and the center of the most distal contact of the implanted electrode (iTP). The coordinates of these points are extracted for QA analysis.

The 3D coordinates extracted as described above are sufficient to calculate the metrics P1 through P5 using formulas of analytic geometry.

\section{Imaging Data}

The anatomically correct (spatial error $<1 \mathrm{~mm}$ ) gadolinium-enhanced T1-weighted MR image (3T GE Discovery 750 scanner, fast spoiled gradient echo, 3D acquisition, TR $7.372 \mathrm{msec}$, TE $3.056 \mathrm{msec}$, flip angle $11^{\circ}$, matrix $256 \times 256$, slice thickness $1 \mathrm{~mm}$, FOV $256 \times 256 \mathrm{~mm}^{2}$ ) was used for planning. The navigation and postimplantation CT images were acquired using the same protocol (Siemens SOMATOM Definition AS+, $120 \mathrm{kVp}$, pixel size $0.42 \times 0.42 \mathrm{~mm}^{2}$, slice thickness $1 \mathrm{~mm}$, gantry tilt $0^{\circ}$ ).

\section{Results}

The descriptive statistics of the accuracy of the three variants of the implantation technique are given in Table 1 . The results are presented using mean and standard deviation metrics to enable comparison between the three variations of the technique, because only these statistics are available for the M1 cohort. The detailed analysis of the complete set of parameters describing key factors affecting accuracy was performed for techniques M2 and M3, in which the full set of QA data were available (Fig. 2). The distribution of 5 of 6 parameters is non-Gaussian, which follows from the plot.

The nonparametric Wilcoxon rank-sum test shows a significant difference between cohorts M2 and M3 for each of the parameters, with greater accuracy using technique M3: P1 (lateral shift at EP), $p<0.01$; P2 (lateral shift of pTP from the bolt axis), $\mathrm{p}<0.01$; P3 (angle between the implanted bolt axis and the planned trajectory), $\mathrm{p}<0.01$; P4 (lateral shift of the tip of the implanted electrode from the bolt axis), $p<0.01$; P5 (deviation of the tip of the implanted electrode from the pTP along the planned trajectory); and integral accuracy (distance between the iTP and pTP), $p<0.01$. Figure 2 illustrates a comparison of cohorts M2 and M3 using box plots.

Further analysis of outliers for P4 (Table 2) suggested that electrodes sampling medial and lateral orbitofrontal cortex (Fig. 3, red dots) were the most prone to bending if the stylet is inserted significantly short of the TP. This was observed in the M3 subgroup when the length of the inserted stylet was recorded. The introduction of the stylet to less than the full length of the planned trajectory reflected 
TABLE 1. Descriptive statistics for the accuracy of the three modifications of the frameless implantation technique

\begin{tabular}{ccccccc}
\hline Modification of Implantation Technique & P1, mm & P2, mm & P3, degrees & P4, mm & P5, mm & Distance, mm \\
\hline M1 & NA & $3.8(2.5)$ & NA & $1.7(1.5)$ & $3.0(4.0)$ & $5.7(3.3)$ \\
\hline M2 & $3.0(1.6)$ & $3.2(1.8)$ & $3.5(1.8)$ & $2.0(1.4)$ & $2.3(2.6)$ & $4.5(2.3)$ \\
\hline M3 & $1.4(0.8)$ & $1.6(0.9)$ & $1.1(0.8)$ & $1.5(1.4)$ & $1.6(1.8)$ & $3.2(1.8)$ \\
\hline
\end{tabular}

Distance = TP, iTP; NA = not available; P1 = lateral shift of EP; P2 = trajectory accuracy lateral shift of TP; P3 = angle (plan, bolt); P4 = electrode bending; $\mathrm{P} 5=$ depth deviation.

All data given as mean (SD).

surgical decisions aimed to increase the safety of the procedure and was dictated by several factors (e.g., relative safety of the trajectory, protocol of vascular imaging).

The deviation of the distal contact of the implanted electrode from the axis of the implanted bolt (electrode bending, P4) was significantly correlated with the length of the electrode from its distal tip to the inner skull surface for two subgroups of the electrodes within cohort M3: 63 electrodes when the tip of the stylet was less than $6 \mathrm{~mm}$ (present standard of our practice) from the length of the planned trajectory $(R=0.45, p<0.01 ;$ Fig. 4$)$ and 46 electrodes when the stylet was placed more than $10 \mathrm{~mm}$ short of the target $(\mathrm{R}=0.67, \mathrm{p}<0.01)$.

The angle between the implanted bolt and the skull surface was positively correlated $(\mathrm{R}=0.2, \mathrm{p}=0.006)$ with the inaccuracy of the angle (bolt axis, plan; parameter P3) but not with the lateral deviation of the iEP (parameter P1) in cohort M3 in which the angle to the skull was measured (Fig. 5).

A mixed-effects linear model using the outcome variables of the angle between the bolt axis and the skull surface, and the angle between the bolt axis and planned trajectory, was fitted along with the patient-level random effect included to account for the clustering of electrodes within patients. This model showed a significant positive linear relationship between the outcome variables.

The duration of drilling the skull to make the hole for inserting the bolt (measured in the M3 cohort, $\mathrm{n}=16$ patients, 148 trajectories; median 30 seconds, IQR1/IQR3 $=22 / 50$ seconds) was not correlated with parameters P1,

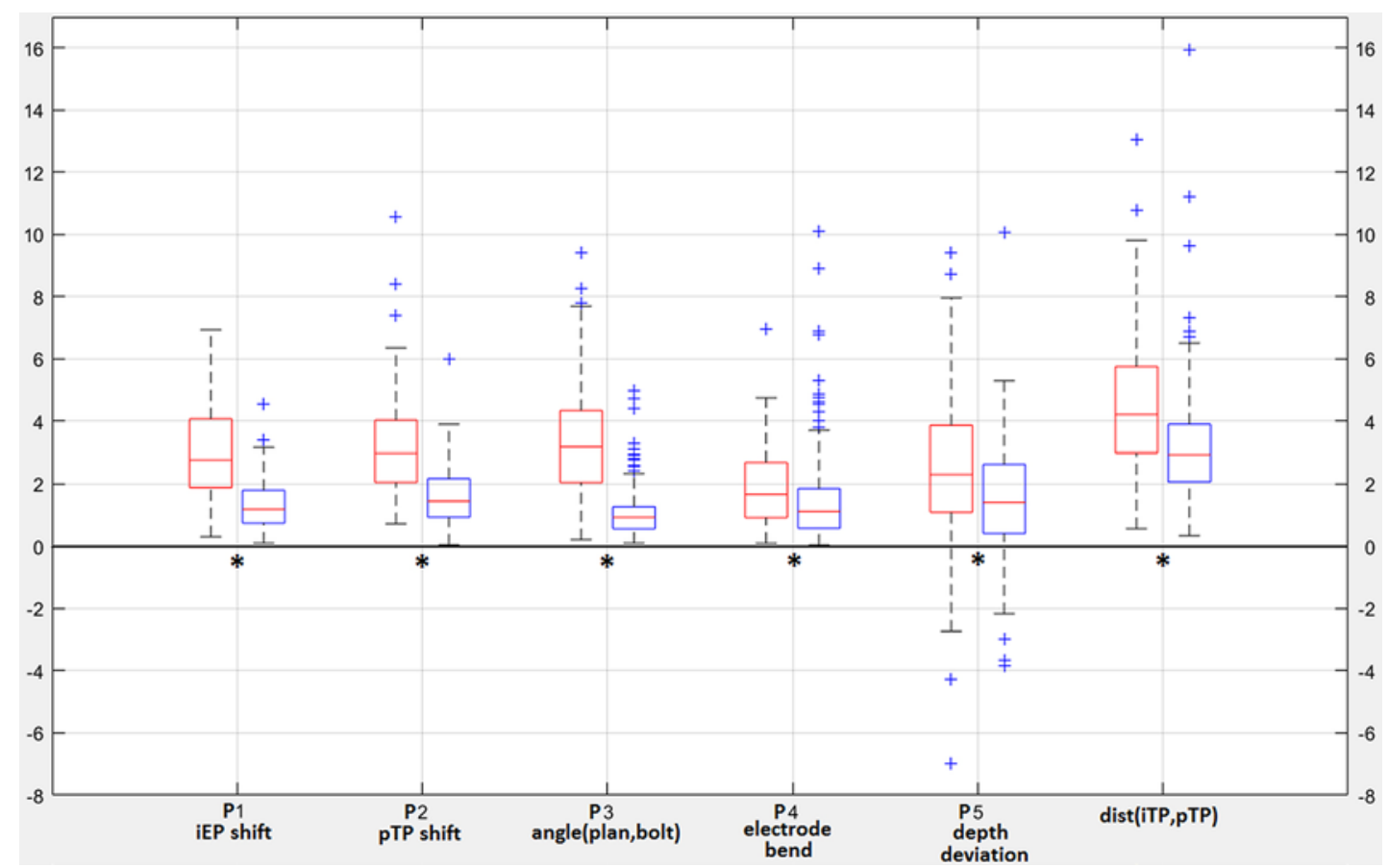

FIG. 2. Summary statistics (median, IQR, minimum/maximum, and outliers) for 6 parameters describing the M2 (red bars) and M3 (blue bars) techniques: P1 (lateral shift of iEP from planned trajectory), P2 (lateral shift of pTP from bolt axis), P3 (angle between planned trajectory and bolt axis), P4 (lateral shift of iTP from bolt axis assessing the amount of bending), P5 (deviation to the depth along the planned trajectory), and integral parameter (distance between iTP and pTP). Vertical axis = mm, except for P3 (degrees). $+=$ outliers. * Statistical significance (unpaired Wilcoxon rank-sum test) of the difference for the corresponding pair. Whiskers = either minimum/maximum value in the corresponding sample if there are no outliers, or limits of the range at $Q 3+1.5 \times I Q R$ or Q1 - 1.5 x IQR when there are values beyond these limits. IQR = Q3 (75th percentile) - Q1 (25th percentile). The limits marked by whiskers are analogous to approximately \pm 2.7 (SD) and $99.3 \%$ coverage if the data were normally distributed. Figure is available in color online only. 
TABLE 2. Analysis of the outliers shown in Fig. 2

\begin{tabular}{lcc}
\hline Parameter & No. of Outliers & Factor Explaining Majority of the Outliers (no. of such outliers) \\
\hline P1 & 2 & $\begin{array}{c}\text { Anterior temporal lobe electrodes (amygdala, anterior hippocampus, temporal pole) where the } \\
\text { skull bone is thin, and so unsuitable for stable fixation of the bolt }(n=2)\end{array}$ \\
\hline P2 & 4 & $\begin{array}{c}\text { Significant deviation of the angle (plan, bolt; P3), or significant deviation of the iEP (P1), or both } \\
\text { of these parameters }(n=4)\end{array}$ \\
\hline P3 & 14 & Anterior temporal lobe electrodes $(n=8)$ \\
\hline P4 & 12 & $\begin{array}{c}\text { Stylet was inserted } 20-35 \text { mm short of the pTP }(n=8) ; \text { frontal lobe electrodes where electrodes } \\
\text { traversed dense white matter tracts en route to the pTP }(n=10)\end{array}$ \\
\hline Distance (iTP, pTP) & 8 & No systematic factor observed \\
\hline
\end{tabular}

$\mathrm{P} 2$, and $\mathrm{P} 3$. This rejects the hypothesis that deviations are more likely when the surgeon has to remain very stable for a prolonged time.

\section{Discussion}

We report our experience of evolving SEEG electrode frameless implantation techniques, based on the results of the QA process established for monitoring implantation accuracy. We used two groups of patients that were defined based on the utilized surgical technique to demonstrate the application of the QA process and its benefits. The results illustrate that the detailed quantification of implantation accuracy informs the modification of the stages of the technique.

The main findings of our work are:

- The accuracy of the technique of frameless implanta- tion has the potential to approach the accuracy of robot-aided techniques, while the high reproducibility of accurate implantation remains a significant advantage when using robots.

- The inaccuracies due to bending and depth deviation are significant contributors to the inaccuracy of electrode tip placement.

- The average accuracy of the electrodes inserted through anterior temporal bone is less than other areas of skull, where the bone is thicker.

- There is a significant correlation between the drill-toskull angle and the accuracy of the implemented trajectory, with higher accuracy when the planned trajectory is close to orthogonal to the bone.

- Advancing the stylet close to the pTP reduces electrode bending.

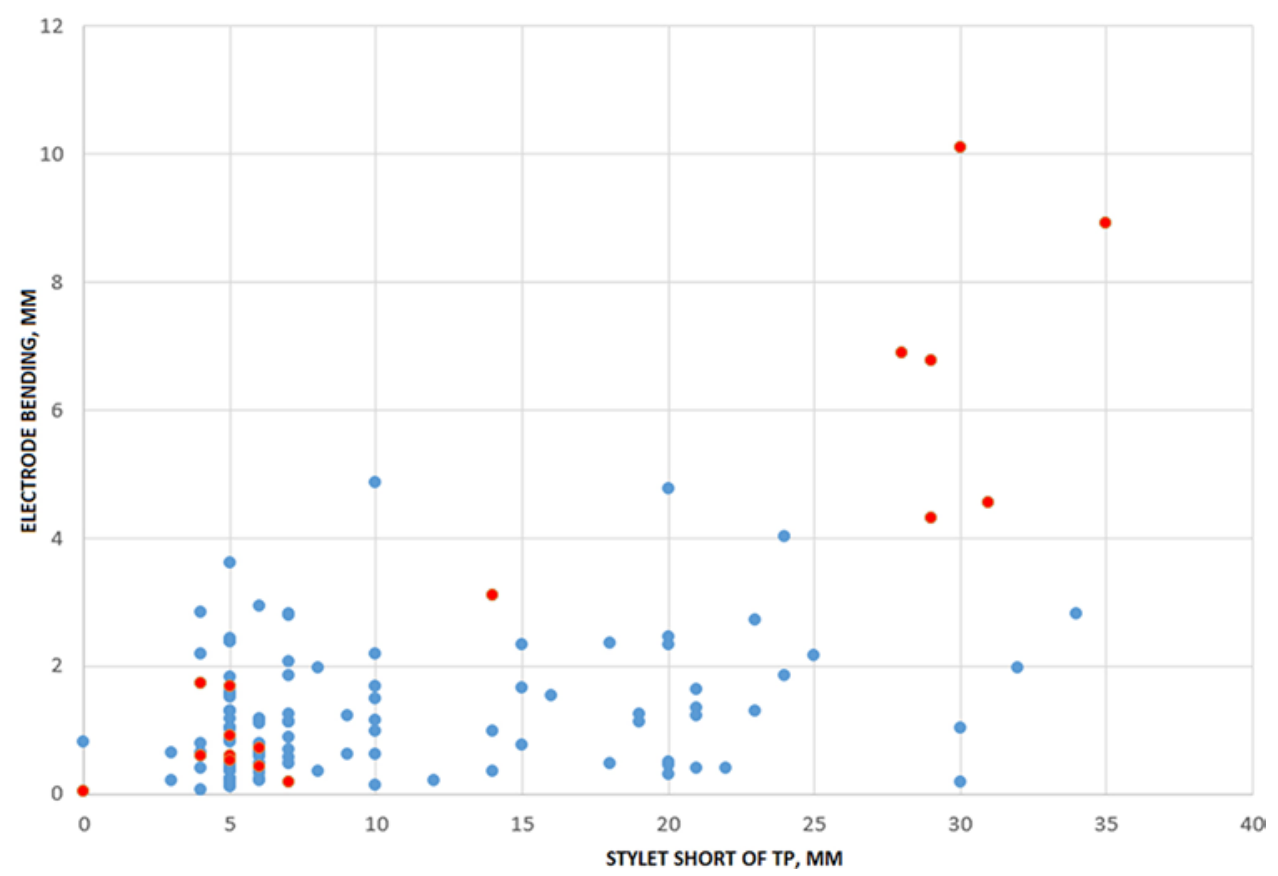

FIG. 3. Graph showing the relationship between bending of the electrodes (parameter P4, vertical axis, $\mathrm{mm}$ ) and distance that the inserted stylet was short of the pTP (horizontal axis, $\mathrm{mm}$ ), based on 129 recorded values. Red dots = electrodes sampling lateral and middle orbitofrontal cortex $(R=0.91, p<0.01)$; blue dots = all other electrodes. Figure is available in color online only. 


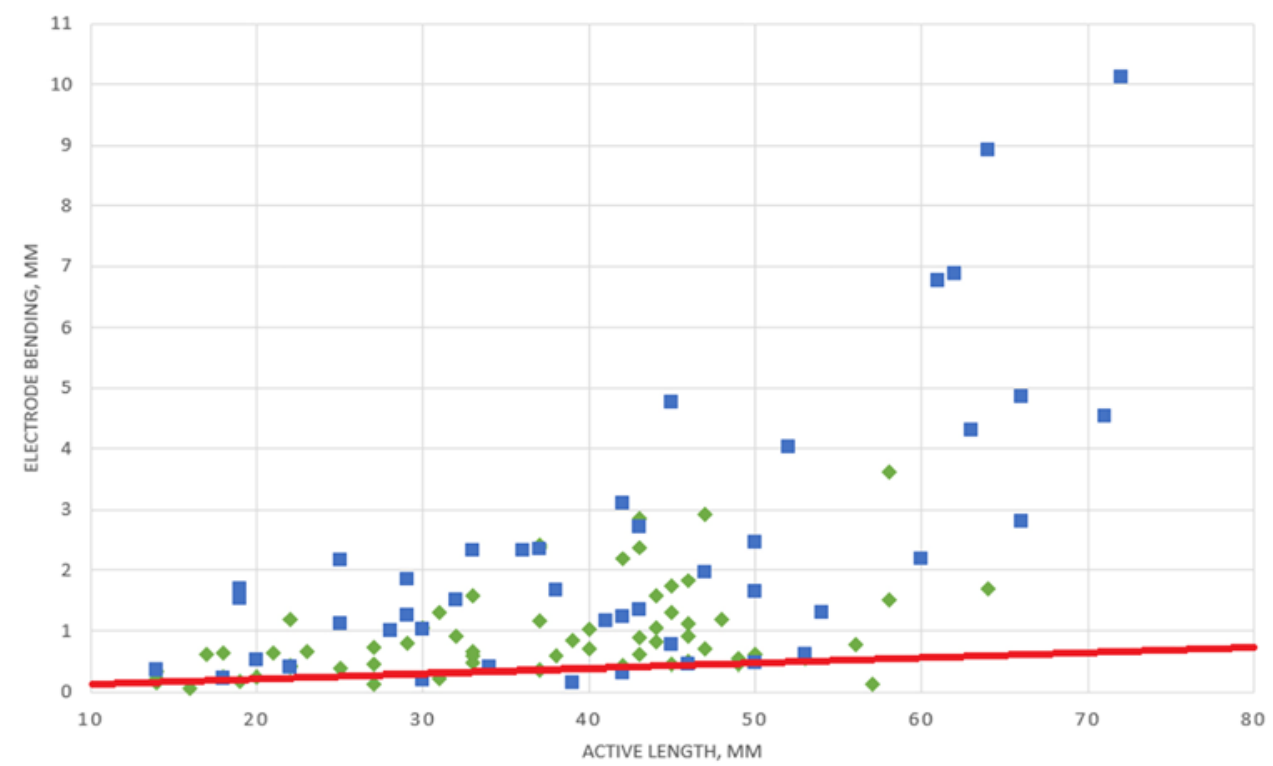

FIG. 4. Graph showing the relationship between the intracranial length of the implanted electrode (horizontal axis, mm) and the electrode bending measured at its distal contact (parameter P4, vertical axis, $\mathrm{mm}$ ) for 63 electrodes when the stylet was advanced close to the TP (less than $6 \mathrm{~mm}$; green rhomboids) and 46 electrodes when the stylet was more than $10 \mathrm{~mm}$ from the TP (b/ue squares) in the M3 cohort. Red line = maximal possible contribution of the stylet deviating from the bolt axis due to wobble of the stylet within the bolt. Figure is available in color online only.

- The detailed QA is useful for monitoring the accuracy of the SEEG electrode implantation procedure and isolating the factors affecting accuracy.

The majority of the electrodes were implanted with an accuracy that is sufficient to sample from typical targets such as the hippocampus (Fig. 2). The median and IQR values of accuracy at the EP and TP in the M3 cohort were similar to those achieved using a novel implantation approach based on the individualized frame. ${ }^{11}$ Unsurpris- ingly, they were inferior to the accuracy achieved using robot-guided implantation, a key feature of which is an outstanding stability of the drill in relation to the patient's head. ${ }^{1}$

The improvement in accuracy is evident (Table 1, Fig. 2). The highly significant increase in accuracy implementing the trajectory was expected as the difference between techniques M1, M2, and M3 is limited to this stage. Ongoing QA data (not presented) show that the accuracy of bolt

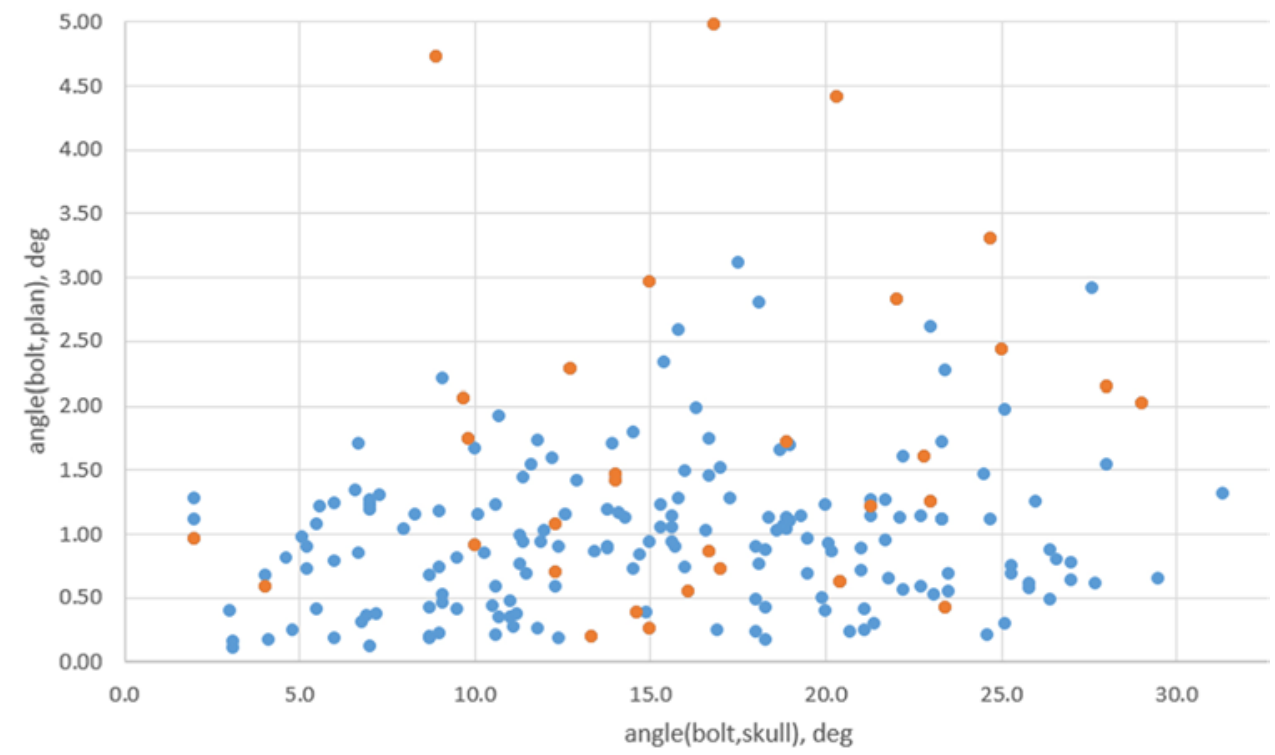

FIG. 5. Relationship between the bolt axis and the planes' trajectory (vertical axis) and between the bolt axis and the skull surface (horizontal axis) in the M3 cohort. Orange dots = anterior temporal lobe electrodes; blue dots = all other electrodes. Figure is available in color online only. 
placement has leveled off since the technique of the M3 cohort was established. Further improvements are possible with modification of the hardware used for bolt placement, including robotic guidance. ${ }^{2}$

The accuracy of the placement of the electrode tip is dependent on the depth to which the stylet is introduced and the degree of electrode bending. With increasing confidence in the ability to avoid blood vessels, the stylet is advanced to the actual electrode target. A further possible modification is to introduce a technique of predicting the direction and amount of electrode bending. ${ }^{4}$

Analysis of outliers leads to important recommendations:

- Inaccuracy may be introduced during electrode insertion, which mandates consideration of modification of the technique for electrode insertion.

- Bolts placed in the anterior temporal bone are susceptible to inaccuracy, and these bolts may be dislodged after insertion because of the thin overlying skull bone.

- The use of stylet-to-target as opposed to shortened stylet insertions enhances the accuracy of the electrode tip and can be adopted if there is confidence in the ability to avoid compromising blood vessels.

The drill bit is likely to slip on the skull surface when the angle is less than $65^{\circ}-70^{\circ}$. This effect depends on the characteristics of the drill bit. A hard steel bit, which is less prone to bending, and a flat profile of the tip are factors that will aid in increased accuracy of implementing the trajectory, even in the event of a low angle to the skull.

The duration of drilling to implement the planned trajectory did not correlate with the accuracy of bolt placement. This observation relates to a single surgeon who performed drilling for all trajectories in this report. This may not hold true for other surgeons and thus should be borne in mind when implementing the technique. We suggest considering measures to increase the stability when drilling (e.g., using arm supports and a handpiece-type drill).

The analysis of outliers points to lower accuracy of placement of anterior temporal lobe electrodes. This phenomenon is widely acknowledged by surgeons performing SEEG electrode implantation. The causes of the reduced accuracy are poor stability due to lack of the support for the bolt due to a very thin temporal bone plate of 1- to $1.5-\mathrm{mm}$ thickness in some cases, and with a thick layer of overlying muscle.

We subsequently tested the hypothesis that implanted bolts can be displaced following implantation. We identified 6 patients who had a clinical need to have two CT images obtained following the implantation (63 electrodes; 8 anterior temporal electrodes across 5 patients). Only the anterior hippocampal bolts were displaced during the time span of a few days between the two CT scans in 2 of 5 patients who had anterior temporal lobe electrodes. All extratemporal bolts remained stable. This observation supports two hypotheses: 1) our model of implantation accuracy for bolt placement is valid apart from the cases targeting anterior temporal lobe structures through the thin temporal bone; and 2) separating the anterior temporal electrodes is reasonable for the statistical analysis of bolt placement accuracy.

The presented QA framework is capable of supporting decision-making about safety and the possibility of implementing specific planned trajectories using a method assessed with this QA. For this purpose, the safety margins for the metrics of the lateral shifts can be derived from the QA data performed for a specific implantation technique. The safety margins are then applied to the planned trajectory in question to assess risk of vascular damage and missing the desired target when using the specific technique. The safety margins would be defined based on the rate of significant deviations of accuracy that the operator considers acceptable. Inevitably, the longer the trajectory, the greater is the possible error at the TP. In this way, it will be possible to choose an optimal technique for long and difficult trajectories, such as longitudinal hippocampal electrodes that have become popular with the introduction of laser interstitial thermal therapy of hippocampal sclerosis.

The influence of the observer effect on the presented metrics of implantation accuracy is possible. We were not able to assess this effect as the QA process is applied continuously to all procedures.

Our frameless implantation technique is a low-cost alternative to robot-guided implantation with drawbacks of lower accuracy (larger average values of the metrics and wider standard deviations) and longer duration of the operation. The latter is a function of the quality of the articulated arm used to align the drill guide with the plan and experience of the operating surgeon. A detailed comparison of our technique with a robot-guided method is the subject of a randomized controlled trial that we are currently undertaking.

\section{Conclusions}

Our QA framework helped to identify the nature and extent of inaccuracies in SEEG implantation, and to monitor ongoing accuracy and the effect of technique modifications.

\section{Acknowledgments}

We are grateful for the enthusiastic participation of the NHNN epilepsy surgery team. This work was supported by a Wellcome Trust grant (no. 106882). R. Rodionov, M. Nowell, and V. N. Vakharia were supported by the Wellcome Trust grant.

\section{References}

1. Cardinale F, Cossu M, Castana L, Casaceli G, Schiariti MP, Miserocchi A, et al: Stereoelectroencephalography: surgical methodology, safety, and stereotactic application accuracy in 500 procedures. Neurosurgery 72:353-366, 2013

2. Dorfer C, Minchev G, Czech T, Stefanits H, Feucht M, Pataraia $\mathrm{E}$, et al: A novel miniature robotic device for frameless implantation of depth electrodes in refractory epilepsy. J Neurosurg 126:1622-1628, 2017

3. González-Martínez J, Bulacio J, Thompson S, Gale J, Smithason S, Najm I, et al: Technique, results, and complications related to robot-assisted stereoelectroencephalography. Neurosurgery 78:169-180, 2016

4. Granados A, Vakharia V, Rodionov R, Schweiger M, Vos SB, O'Keeffe AG, et al: Automatic segmentation of stereoelectroencephalography (SEEG) electrodes post-implantation considering bending. Int J CARS 13:935-946, 2018

5. Mascott CR: In vivo accuracy of image guidance performed 
using optical tracking and optimized registration. J Neurosurg 105:561-567, 2006

6. Munyon CN, Koubeissi MZ, Syed TU, Lüders HO, Miller JP: Accuracy of frame-based stereotactic depth electrode implantation during craniotomy for subdural grid placement. Stereotact Funct Neurosurg 91:399-403, 2013

7. Nowell M, Rodionov R, Diehl B, Wehner T, Zombori G, Kinghorn J, et al: A novel method for implementation of frameless stereoEEG in epilepsy surgery. Neurosurgery 10 (Suppl 4):525-534, 2014

8. Ortler M, Sohm F, Eisner W, Bauer R, Dobesberger J, Trinka E, et al: Frame-based vs frameless placement of intrahippocampal depth electrodes in patients with refractory epilepsy: a comparative in vivo (application) study. Neurosurgery 68:881-887, 2011

9. Vakharia VN, Sparks R, Rodionov R, Vos SB, Dorfer C, Miller J, et al: Computer-assisted planning for the insertion of stereoelectroencephalography electrodes for the investigation of drug-resistant focal epilepsy: an external validation study. J Neurosurg 13:1-10, 2018

10. Verburg N, Baayen JC, Idema S, Klitsie MA, Claus S, de Jonge CS, et al: In vivo accuracy of a frameless stereotactic drilling technique for diagnostic biopsies and stereoelectroencephalography depth electrodes. World Neurosurg 87:392-398, 2016

11. Yu H, Pistol C, Franklin R, Barborica A: Clinical accuracy of customized stereotactic fixtures for stereoelectroencephalography. World Neurosurg 109:82-88, 2018

\section{Disclosures}

Collaborative support was received from Medtronic Ltd in the form of an S7 StealthStation planning computer (on loan).

\section{Author Contributions}

Conception and design: Rodionov, Duncan. Acquisition of data: Rodionov, Nowell, Rizzi, Vakharia, Wykes, Miserocchi, McEvoy. Analysis and interpretation of data: Rodionov, Nowell, Rizzi, Duncan. Drafting the article: Rodionov. Critically revising the article: all authors. Reviewed submitted version of manuscript: Rodionov, Nowell, Rizzi, Vakharia, Wykes, Eriksson, Miserocchi, McEvoy, Ourselin, Duncan. Approved the final version of the manuscript on behalf of all authors: Rodionov. Statistical analysis: Rodionov, O'Keeffe. Administrative/technical/material support: Eriksson. Study supervision: Duncan.

\section{Supplemental Information}

Online-Only Content

Supplemental material is available with the online version of the article.

Supplementary Material. https://thejns.org/doi/suppl/10.3171/ 2019.2.JNS183313.

\section{Correspondence}

Roman Rodionov: UCL Queen Square Institute of Neurology, University College London, United Kingdom. roman.rodionov@ gmail.com. 